\title{
Calibration of the Ørsted vector magnetometer
}

\author{
Nils Olsen ${ }^{1}$, Lars Tøffner-Clausen ${ }^{2}$, Terence J. Sabaka ${ }^{3}$, Peter Brauer ${ }^{4}$, Jose M. G. Merayo ${ }^{4}$, John L. Jørgensen ${ }^{4}$, \\ J.-M. Léger ${ }^{5}$, Otto V. Nielsen ${ }^{4}$, Fritz Primdahl ${ }^{1,4}$, and Torben Risbo ${ }^{6}$ \\ ${ }^{1}$ Danish Space Research Institute, Juliane Maries Vej 30, 2100 Copenhagen Ø, Denmark \\ ${ }^{2}$ Ørsted Science Data Center, DMI, Lyngbyvej 100, 2100 Copenhagen Ø, Denmark \\ ${ }^{3}$ Raytheon ITSS, Lanham, MD 20706, U.S.A. \\ ${ }^{4}$ Oersted.DTU, Technical University of Denmark, Building 327, 2800 Lyngby, Denmark \\ ${ }^{5}$ CEA-Direction des Technologies Avancées, France \\ ${ }^{6}$ Niels Bohr Institute AFG, Juliane Maries Vej 30, 2100 Copenhagen Ø, Denmark
}

(Received July 1, 2002; Revised November 14, 2002; Accepted November 26, 2002)

\begin{abstract}
The vector fluxgate magnetometer of the Ørsted satellite is routinely calibrated by comparing its output with measurements of the absolute magnetic intensity from the Overhauser instrument, which is the second magnetometer of the satellite. We describe the method used for and the result obtained in that calibration. Using three years of data the agreement between the two magnetometers after calibration is $0.33 \mathrm{nT} \mathrm{rms} \mathrm{(corresponding} \mathrm{to} \mathrm{better} \mathrm{than}$ $\pm 1 \mathrm{nT}$ for $98 \%$ of the data, and better than $\pm 2 \mathrm{nT}$ for $99.94 \%$ of the data). We also report on the determination of the transformation between the magnetometer coordinate system and the reference system of the star imager. This is done by comparing the magnetic and attitude measurements with a model of Earth's magnetic field. The Euler angles describing this rotation are determined in this way with an accuracy of better than 4 arcsec.
\end{abstract}

\section{Introduction}

The Ørsted satellite was launched on February 23, 1999 in a near polar orbit with an inclination of $96.5^{\circ}$, a perigee at $640 \mathrm{~km}$ and an apogee at $880 \mathrm{~km}$. The principal aim of the Ørsted mission (Neubert et al., 2001) is to accurately map the Earth's magnetic field (Olsen et al., 2000a, b; Olsen, 2002), and high-precision magnetic field measurements spanning three years are now available. The satellite is equipped with a scalar Overhauser magnetometer $(\mathrm{OVH})$ (Duret et al., 1996), a tri-axial fluxgate magnetometer (CSC, for Compact Spherical Coil) (Nielsen et al., 1995, 1997) and a Star Imager (SIM) (Jørgensen, 2000) which determines the attitude of the satellite with high accuracy. The OVH is mounted at the top of an $8 \mathrm{~m}$ long boom; the CSC and the SIM are mounted closely together on an optical bench which is located $2 \mathrm{~m}$ away from the scalar magnetometer and $6 \mathrm{~m}$ away from the satellite body. The magnetic field measured by the Overhauser instrument is assumed to be unaffected by space-craft fields, due to the larger distance to the space-craft ( $8 \mathrm{~m}$ for the OVH compared to $6 \mathrm{~m}$ for the CSC reduces the space-craft fields (to first order) by a factor of $(8 / 6)^{3} \approx 2.4$ at the location of the $\mathrm{OVH}$ instrument).

The CSC magnetometer is designed to be a highly stable, linear instrument. However, as typical for fluxgate magnetometers it is not an absolute instrument, and hence it has to be calibrated (see Lancaster et al. (1980) for a description of the magnetometer calibration of Magsat, the first and only high-precision vector field satellite before Ørsted, and

Copy right(c) The Society of Geomagnetism and Earth, Planetary and Space Sciences (SGEPSS); The Seismological Society of Japan; The Volcanological Society of Japan; The Geodetic Society of Japan; The Japanese Society for Planetary Sciences.
Gödderz et al. (2002) for a description of the calibration of the CHAMP magnetometer). In addition, the relative rotation between the coordinate system of the CSC and that of the SIM has to be determined with an accuracy of a few arcseconds. Estimation of the three Euler angles (which describe the rotation) is also called alignment of the magnetometer (Merayo et al., 2001; Jørgensen et al., 2001). In the following we will use the term calibration for the estimation of the magnetometer response as well as of the Euler angles.

The CSC magnetometer has been calibrated and aligned before launch (pre-flight calibration). However, in-flight estimation of the calibration parameters is performed routinely. In this paper we focus on the methods used and the results obtained with in-flight calibration.

There are two different approaches for calibrating a magnetometer: In a vector calibration the output of the vector magnetometer (to be calibrated) is compared with the (known) magnetic field vector that is applied to the instrument. Contrary to this approach, only the scalar intensity of the magnetic field (but not its direction) is used in a scalar calibration.

Clearly, a vector calibration is superior to a scalar calibration if the magnetic field vector is known. If it is only known approximately (for instance by assuming that it can be approximated by a model of the Earth's magnetic field), a scalar calibration is superior for the estimation of the magnetometer response (scale values, offsets and non-orthogonalities). Scalar intensity is routinely measured by the Overhauser scalar magnetometer, and therefore a scalar calibration is straightforward.

However, with scalar data alone it is not possible to esti- 


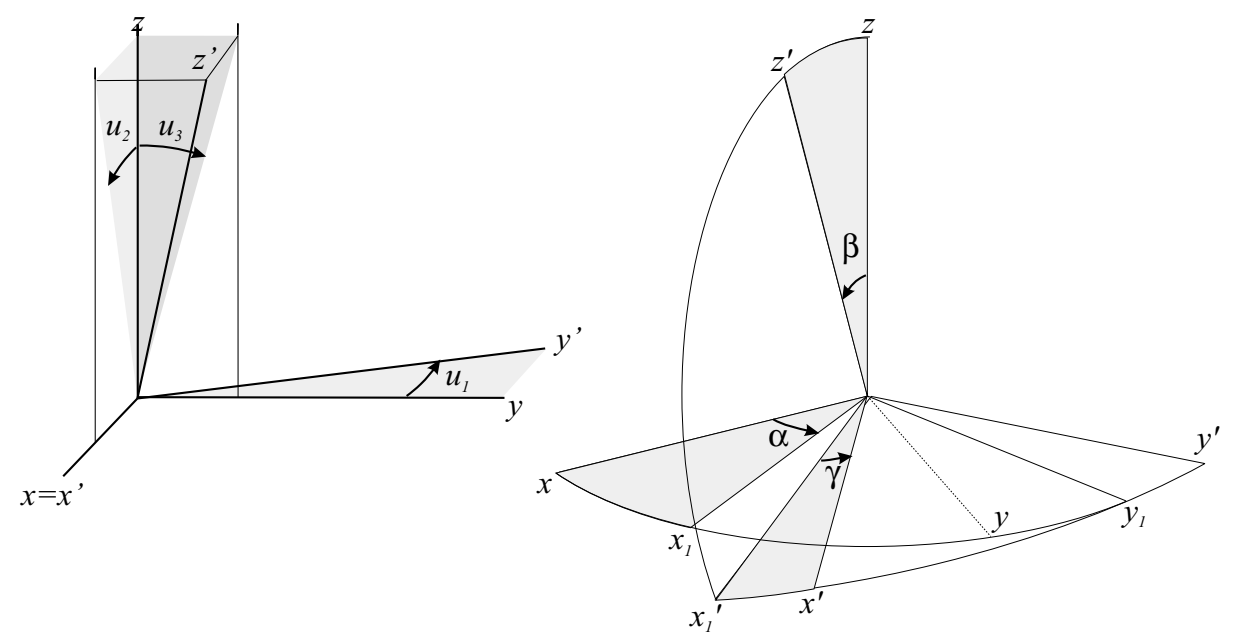

Fig. 1. Left: Definition of the non-orthogonalities $\left(u_{1}, u_{2}, u_{3}\right)$. The matrix $\underline{\mathbf{P}}$ of Eq. (2) transforms a vector $\mathbf{B}$ whose components are given in the orthogonal coordinate system to the vector $\mathbf{B}^{\prime}=\underline{\mathbf{P}} \cdot \mathbf{B}$ whose components are given in the (primed) non-orthogonal coordinate system. Right: Definition of the Euler angles $(\alpha, \beta, \gamma)$ (e.g., equation (11.6) of Synge, 1960).

mate the rotation between the magnetometer coordinate system and the reference system, which is the coordinate system of the SIM in the case of Ørsted. Determination of this rotation is performed by comparing the measurements of the magnetic field vector and of the attitude with an independently determined model (Olsen, 2002) of the Earth's magnetic field.

The content of the paper is as follows: in Section 2 we discuss the response of a linear magnetometer. Estimation of the parameters of this response by means of an in-flight scalar calibration is the topic of the third section, and the fourth section deals with the estimation of the rotation matrix (Euler angles) between the CSC and SIM coordinate systems.

\section{The Response of a Linear Vector Magnetometer}

Pre-flight calibrations have demonstrated that the Ørsted vector magnetometer is a linear instrument (Merayo et al., 2000; Risbo and Olsen, 1996; Risbo et al., 2002), which means that the magnetometer output $\mathbf{E}=\left(E_{1}, E_{2}, E_{3}\right)^{T}$ (in engineering units, eu) is connected to the applied magnetic field vector $\mathbf{B}_{\mathrm{CSC}}=\left(B_{1}, B_{2}, B_{3}\right)^{T}$ (in the orthogonal magnetic axes system) according to

$$
\mathbf{E}=\underline{\underline{\mathbf{S}}} \cdot \underline{\underline{\mathbf{P}}} \cdot \mathbf{B}_{\mathrm{CSC}}+\mathbf{b}
$$

where

$$
\mathbf{b}=\left(\begin{array}{l}
b_{1} \\
b_{2} \\
b_{3}
\end{array}\right)
$$

is the vector of offsets (in eu),

$$
\underline{\underline{\mathbf{S}}}=\left(\begin{array}{lll}
S_{1} & 0 & 0 \\
0 & S_{2} & 0 \\
0 & 0 & S_{3}
\end{array}\right)
$$

is the (diagonal) matrix of sensitivities (in eu/nT), and

$$
\underline{\mathbf{P}}=\left(\begin{array}{ccc}
1 & 0 & 0 \\
-\sin u_{1} & \cos u_{1} & 0 \\
\sin u_{2} & \sin u_{3} & \sqrt{\left(1-\sin ^{2} u_{2}-\sin ^{2} u_{3}\right)}
\end{array}\right)
$$

is a matrix which transforms a vector from the orthogonal magnetic axes coordinate system to the non-orthogonal magnetic sensor axes coordinate system. Magnetometer axis 1 is assumed to be projection invariant, axis 2 has a single degree of freedom in the 1-2 plane, and magnetometer axis 3 has two degrees of freedom. The projection consists of three angles, the first being between the orthogonal and nonorthogonal axes 2 , and the second and third being between the orthogonal and non-orthogonal axes 3 . The third magnetometer axis is about $30^{\circ}$ off the boom direction (pointing towards the space-craft); the second axis is perpendicular to the boom direction, and the third axis completes a right-hand coordinate system. The sensitivity matrix, $S$, is assumed to be diagonal. This means that all the cross-talk components between axes are zero, which has been confirmed by the pre-flight calibration (Risbo et al., 2002). The left part of Fig. 1 shows the geometry used for this definition of the non-orthogonalities.

The 9 parameters $b_{i}, S_{i}, u_{i}, i=1,2,3$ completely describe the response of a linear magnetometer (some of the parameters, however, may depend on temperature and on time). Three additional parameters, the Euler angles $\alpha, \beta, \gamma$, are necessary to transform the magnetic field vector $\mathbf{B}_{\mathrm{SIM}}$ in the SIM coordinate system to the vector

$$
\mathbf{B}_{\mathrm{CSC}}=\underline{\underline{\mathbf{R}}} \cdot \mathbf{B}_{\mathrm{SIM}}
$$

in the magnetometer system. We describe this transformation by a "3-2-3" rotation (e.g., equation (11.6) of Synge, 
1960) given by

$$
\begin{aligned}
\underline{\underline{\mathbf{R}}}= & \left(\begin{array}{ccc}
\cos \alpha & -\sin \alpha & 0 \\
\sin \alpha & \cos \alpha & 0 \\
0 & 0 & 1
\end{array}\right)\left(\begin{array}{ccc}
\cos \beta & 0 & \sin \beta \\
0 & 1 & 0 \\
-\sin \beta & 0 & \cos \beta
\end{array}\right)\left(\begin{array}{ccc}
\cos \gamma & -\sin \gamma & 0 \\
\sin \gamma & \cos \gamma & 0 \\
0 & 0 & 1
\end{array}\right) \\
= & \left(\begin{array}{cc}
\cos \alpha \cos \beta \cos \gamma-\sin \alpha \sin \gamma \\
\sin \alpha \cos \beta \cos \gamma+\cos \alpha \sin \gamma \\
-\sin \beta \cos \gamma \\
-\cos \alpha \cos \beta \sin \gamma-\sin \alpha \cos \gamma & \cos \alpha \sin \beta \\
-\sin \alpha \cos \beta \sin \gamma+\cos \alpha \cos \gamma & \sin \alpha \sin \beta \\
\sin \beta \sin \gamma & \cos \beta
\end{array}\right)
\end{aligned}
$$

(cf. the right part of Fig. 1). Hence the connection between the sensor output $\mathbf{E}$ and the magnetic field $\mathbf{B}_{\text {SIM }}$ in the reference coordinate system of the SIM is given by

$$
\mathbf{E}=\underline{\underline{\mathbf{S}}} \cdot \underline{\underline{\mathbf{P}}} \cdot \underline{\underline{\mathbf{R}}} \cdot \mathbf{B}_{\mathrm{SIM}}+\mathbf{b} .
$$

Once the calibration parameters are known, the magnetic field in the CSC and SIM coordinate system, respectively, can be determined from the sensor output according to

$$
\begin{aligned}
\mathbf{B}_{\mathrm{CSC}} & =\underline{\underline{\mathbf{P}}}^{-1} \cdot \underline{\underline{\mathbf{S}}}^{-1} \cdot(\mathbf{E}-\mathbf{b}) \\
\mathbf{B}_{\mathrm{SIM}} & =\underline{\underline{\mathbf{R}}}^{-1} \cdot \mathbf{B}_{\mathrm{CSC}} \\
& =\underline{\underline{\mathbf{R}}}^{-1} \cdot \underline{\underline{\mathbf{P}}}^{-1} \cdot \underline{\underline{\mathbf{S}}}^{-1} \cdot(\mathbf{E}-\mathbf{b})
\end{aligned}
$$

where $\underline{\underline{\mathbf{R}}}^{-1}=\underline{\underline{\mathbf{R}}}^{T}$ and

$$
\underline{\underline{P}}^{-1}=\left(\begin{array}{ccc}
1 & 0 & 0 \\
\frac{\sin u_{1}}{\cos u_{1}} & \frac{1}{\cos u_{1}} & 0 \\
w \cos u_{1} & -\frac{\sin u_{3}}{w \cos u_{1}} & \frac{1}{w}
\end{array}\right)
$$

with $w=\sqrt{1-\sin ^{2} u_{2}-\sin ^{2} u_{3}}$.

As already mentioned before, some of the parameters may depend on the temperature of the sensor, $T_{S}$, and on the temperature of the electronics, $T_{A}$. Laboratory measurements and pre-flight calibrations have shown that the sensitivities $S_{i}$ and offsets $b_{i}$ depend linearly on temperature, and that the non-orthogonalities (as well as the rotation between the CSC and the SIM coordinate systems) are temperature independent. We also included an explicit linear dependency on time $t$ (measured in years after January 1, 2000) for the offsets and scale values, to allow for aging effects. Hence scale-value and offset of the $i$ th axis are modeled by respectively

$$
\begin{aligned}
S_{i} & =S_{0, i}+S_{A, i} T_{A}+S_{S, i} T_{S}+S_{t, i} t \\
b_{i} & =b_{0, i}+b_{A, i} T_{A}+b_{t, i} t .
\end{aligned}
$$

(The dependence of the offsets on sensor temperature is negligible, cf. Nielsen et al. (1995). This pre-flight result has been confirmed in orbit.) It follows from Eq. (5) that the scalar intensity $B_{\mathrm{CSC}}$ of the CSC magnetometer as a function of the sensor output $\mathbf{E}$ is given by

$$
\begin{aligned}
B_{\mathrm{CSC}} & =\left|\mathbf{B}_{\mathrm{CSC}}\right|=\sqrt{\mathbf{B}_{\mathrm{CSC}}^{T} \cdot \mathbf{B}_{\mathrm{CSC}}} \\
& =\sqrt{(\mathbf{E}-\mathbf{b})^{T} \cdot \underline{\underline{\mathbf{S}}}^{-1} \cdot\left(\underline{\underline{\mathbf{P}}}^{-1}\right)^{T} \cdot \underline{\underline{\mathbf{P}}}^{-1} \cdot \underline{\underline{\mathbf{S}}}^{-1} \cdot(\mathbf{E}-\mathbf{b}) .}
\end{aligned}
$$

The 9 temperature-independent parameters plus the 3 . $5=15$ values describing the dependencies on temperature and time are collected in the model vector $\mathbf{m}=$ $\left(b_{0, i}, S_{0, i}, u_{i}, b_{A, i}, b_{t, i}, S_{A, i}, S_{S, i}, S_{t, i}\right)^{T}$. We used a linearized robust least-squares approach to estimate these parameters. If the data vector predicted from the model, i.e. Eq. (7), is denoted with an explicit parameter dependence, $\mathbf{d}(\mathbf{m})$, then the $k$ th iteration of the least-squares estimator may be written as

$$
\begin{aligned}
\mathbf{m}^{k+1}= & \mathbf{m}^{k}+\delta \mathbf{m}^{k} \\
\delta \mathbf{m}^{k}= & {\left[\left(\underline{\underline{\mathbf{G}}}^{k}\right)^{T} \cdot \underline{\underline{\mathbf{W}}}_{d}^{k} \cdot \underline{\underline{\mathbf{G}}}^{k}+\underline{\underline{\mathbf{W}}}_{p}\right]^{-1} } \\
& \cdot\left[\left(\underline{\underline{\mathbf{G}}}^{k}\right)^{T} \cdot \underline{\underline{\mathbf{W}}}_{d}^{k} \cdot \delta \mathbf{d}^{k}+\underline{\underline{\mathbf{W}}}_{p} \cdot\left(\mathbf{m}^{k}-\mathbf{m}_{p}\right)\right]
\end{aligned}
$$

where $\delta \mathbf{d}^{k}=B_{\mathrm{OVH}}-B_{\mathrm{CSC}}\left(\mathbf{E}, \mathbf{m}^{k}\right)$ is the data residual vector of the $k$ th iteration,

$$
\underline{\underline{\mathbf{G}}}^{k}=\left.\frac{\partial \mathbf{d}(\mathbf{m})}{\partial \mathbf{m}}\right|_{\mathbf{m}=\mathbf{m}^{k}}
$$

is the data kernel matrix, $\underline{\underline{\mathbf{W}}}_{d}^{k}$ is the diagonal data weight matrix, $\mathbf{m}_{p}$ is the a priori model vector, and $\underline{\underline{\mathbf{W}}}_{p}$ is a diagonal matrix with the weights $w_{p}$ that are associated to these $a$ priori values. We used the pre-flight estimates as a priori values and successively relaxed the weights $w_{p}$ to allow the data to modify the pre-flight values. The model estimate of a parameter is entirely determined by the (in-flight) observations if the corresponding a priori weight $w_{p}$ is set to zero (i.e., no influence of the a priori value). The larger the weight, the stronger the influence of the a priori value. This allows us to force the solution to the a priori value, i.e. to apply the $a$ priori value to the data (rather than to estimate it from the in-flight data).

We used Huber weights (Hogg, 1979) to account for outliers (examination of data residuals justifies this choice $a$ posteriori); these weights are collected in the diagonal data weight matrix $\underline{\underline{\mathbf{W}}}_{d}^{k}$. Since the robust weights depend on the data residuals $\delta \mathbf{d}^{k}$, the elements of $\underline{\underline{\mathbf{W}}}_{d}^{k}$ depend on iteration number $k$, too.

\section{Application}

We applied the method to data of three years (March 1999-April 2002) with 5 minutes sampling rate (one sample every 5 minutes). This data set consists of about 170,000 measurements of $B_{\mathrm{OVH}}, E_{1}, E_{2}, E_{3}, T_{A}$ and $T_{S}$, from which the 24 values $b_{0, i}, S_{0, i}, u_{i}, b_{A, i}, b_{t, i}, S_{A, i}, S_{S, i}, S_{t, i}, i=1-3$ are estimated. In the first run we used $w_{p}=0$ for all parameters (i.e., no influence of the a priori values). Achieved rms misfit was $0.33 \mathrm{nT}$. The results of this in-flight calibration, together with the pre-flight values, are listed in Table 1.

There is excellent agreement between the pre- and in-flight estimated non-orthogonalities $u_{i}$ (less than 2.5 arcsec difference). The difference in the offsets $b_{0, i}$ of up to $3.2 \mathrm{eu}$ is probably due to static space-craft fields that were not accounted for in the pre-flight calibration. Note that the preflight satellite tests were not performed in complete flight configuration (engineering- not flight-battery, solar panels not operating, etc.), i.e. the offsets determined pre-flight are not expected to be precise representatives for the in-flight values. 
Table 1. Results of the in-flight (top) and pre-flight calibration (bottom). The in-flight standard deviations are estimated from the scatter of the residuals shown in Figure 3.

\begin{tabular}{|c|c|c|c|}
\hline in-flight & axis 1 & axis 2 & axis 3 \\
\hline$b_{0, i}[\mathrm{eu}]:$ & $-0.02 \pm 0.02$ & $0.02 \pm 0.02$ & $1.12 \pm 0.02$ \\
\hline$S_{0, i}[\mathrm{eu} / \mathrm{nT}]$ & $(1.0011874 \pm 0.8) \cdot 10^{-6}$ & $(0.9969169 \pm 0.9) \cdot 10^{-6}$ & $(0.9955280 \pm 0.4) \cdot 10^{-6}$ \\
\hline$u_{i}[\operatorname{arcsec}]$ & $316.3 \pm 0.2$ & $66.8 \pm 0.2$ & $-42.2 \pm 0.2$ \\
\hline$b_{A, i}\left[\mathrm{eu} /{ }^{\circ} \mathrm{C}\right]$ & $-33.9 \cdot 10^{-3}$ & $30.3 \cdot 10^{-3}$ & $-3.4 \cdot 10^{-3}$ \\
\hline$S_{A, i}\left[\mathrm{eu} /\left(\mathrm{nT}^{\circ} \mathrm{C}\right)\right]$ & $3.4 \cdot 10^{-6}$ & $1.6 \cdot 10^{-6}$ & $3.4 \cdot 10^{-6}$ \\
\hline$S_{S, i}\left[\mathrm{eu} /\left(\mathrm{nT}^{\circ} \mathrm{C}\right)\right]$ & $12.2 \cdot 10^{-6}$ & $9.5 \cdot 10^{-6}$ & $6.3 \cdot 10^{-6}$ \\
\hline$b_{t, i}[\mathrm{eu} / \mathrm{yr}]:$ & 0.37 & 0.32 & 0.09 \\
\hline$S_{t, i}[\mathrm{eu} /(\mathrm{nT} \mathrm{yr})]:$ & $-40 \cdot 10^{-6}$ & $-15 \cdot 10^{-6}$ & $2 \cdot 10^{-6}$ \\
\hline \multicolumn{4}{|l|}{ pre-flight } \\
\hline$b_{0, i}[\mathrm{eu}]$ & -3.23 & -1.60 & 0.82 \\
\hline$S_{0, i}[\mathrm{eu} / \mathrm{nT}]$ & 1.0011815 & 0.9970043 & 0.9955952 \\
\hline$u_{i}[\operatorname{arcsec}]$ & 314.1 & 66.5 & -43.6 \\
\hline$b_{A, i}\left[\mathrm{eu} /{ }^{\circ} \mathrm{C}\right]$ & $-32.0 \cdot 10^{-3}$ & $-29.0 \cdot 10^{-3}$ & $-15.0 \cdot 10^{-3}$ \\
\hline$S_{A, i}\left[\mathrm{eu} /\left(\mathrm{nT}^{\circ} \mathrm{C}\right)\right]$ & $1.94 \cdot 10^{-6}$ & $-3.80 \cdot 10^{-6}$ & $-1.01 \cdot 10^{-6}$ \\
\hline$S_{S, i}\left[\mathrm{eu} /\left(\mathrm{nT}^{\circ} \mathrm{C}\right)\right]$ & $(12.75 \pm 0.06) \cdot 10^{-6}$ & $(9.76 \pm 0.07) \cdot 10^{-6}$ & $(6.54 \pm 0.08) \cdot 10^{-6}$ \\
\hline
\end{tabular}

The materials of the magnetometer sensor coil shells are under different stress and stress change during in-flight temperature cycling. The magnetometer sensor structure is a composite of different materials, the innermost shell has a thermal expansion coefficient of zero, but the material of the outer shells carrying the compensation coils has an expansion coefficient of approximately $30 \mathrm{ppm} /{ }^{\circ} \mathrm{C}$ (Nielsen $e t$ $a l ., 1995,1997)$. The resulting thermal linear expansion of the feedback coils is a graduation from approximately 13 $\mathrm{ppm} /{ }^{\circ} \mathrm{C}$ to $6 \mathrm{ppm} /{ }^{\circ} \mathrm{C}$ (Risbo et al., 2001). The observed dependencies on sensor temperature can be explained with the assumption of isotropic thermal expansion of the coil shells, but with expansion coefficients depending on the radial distance from the inner zero-expansion shell. The reasons for the observed time change of the scale factors may include aging of the material, launch stresses and stress relief between the pre-flight non-vacuum and the in-flight vacuum environment. The most probable cause of the time dependency of the scale values is, however, related to combined aging and energetic particle radiation dose effects on the magnetometer electronics. For reference, a compilation of all the pre-flight calibration results for the flight and for the two flight-spare CSC sensors are given in Risbo et al. (2001).

Pre- and in-flight estimated scale values differ by up to 90 ppm, comparable in size to the in-flight estimated trend over 2-3 years. The dependencies of the scale values on sensor temperature, $S_{S, i}$, is the mean value of two independent preflight determinations; the listed error bounds are calculated from the difference of the two results. Pre- and in-flight estimates of $S_{S, i}$ are in good agreement (though the difference is greater than the formal pre-flight standard deviation), but the reason for the discrepancy in the dependencies on electronic temperature (for instance for the different signs of preand in-flight estimates of $b_{A, 2}$ ) is unknown. Since electronics temperature varies by about $30^{\circ} \mathrm{C}$ (cf. the upper right panel of Fig. 3), knowledge of the correct temperature dependency is essential for a good calibration.

The black curve of Fig. 2 presents the distribution of the residuals $\delta d=B_{\mathrm{OVH}}-B_{\mathrm{CSC}}$ in a Normal probability plot. Gaussian distributed residuals would result in a straight line in this type of plot; the S-shaped curve indicates the existence of outliers and hence confirm the necessity to use a robust approach. $98 \%$ of the residuals are within $\pm 1 \mathrm{nT}$, and $99.94 \%$ are within $\pm 2 \mathrm{nT}$, corresponding to an overall accuracy better than $0.4 \mathrm{nT}$ rms.

To test whether the data are compatible with (or require modification of) the pre-flight estimates, we successively reduced the number of free parameters (i.e., fixed the parameters to their pre-flight estimates by increasing their a priori weights $w_{p}$ ) and investigated the rms misfit. Ignoring a trend in offsets and scale values (which means assuming that they are constant in time) increases the rms misfit by $50 \%$ (from $0.33 \mathrm{nT}$ to $0.49 \mathrm{nT}$ ). The distribution of the corresponding residuals are shown by the blue curve of Fig. 2; only $92 \%$ of the residuals are now within $\pm 1 \mathrm{nT}$. Finally we applied all pre-flight estimates but the offsets (which are influenced by static space-craft fields and therefore not properly determinable during the pre-flight calibration), i.e. we allow the in-flight observations to modify only the offsets. In this case less than $63 \%$ of the residuals are within $\pm 1 \mathrm{nT}$ (red curve in the figure). This shows that the data are not compatible with (at least some of the) pre-flight determined temperature dependencies. However, in-flight a high-correlation exists between the temperature of the electronics and that of the sensor (see Fig. 3 top right), and therefore the in-flight determined individual temperature dependencies are probably correlated, too.

Next we applied the method to 174 data segments (1 


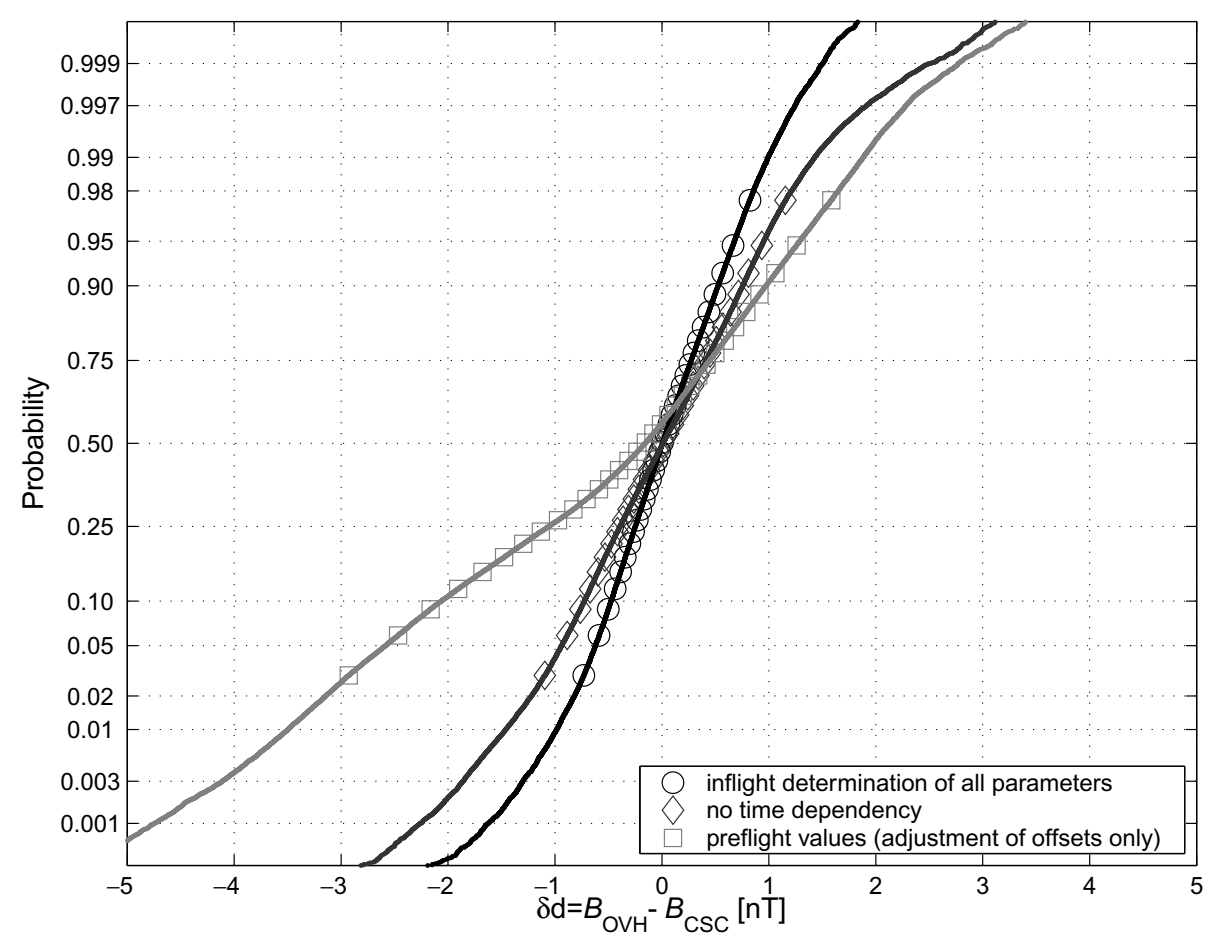

Fig. 2. Normal probability plots of the residuals $\delta d=B_{\mathrm{OVH}}-B_{\mathrm{CSC}}$.

minute sampling rate) of 4-days length, and solved for the 9 parameters $b_{0, i}, S_{0, i}, u_{i}, i=1,2,3$ after applying the inflight temperature dependencies of Table 1 to the data. The left column of Fig. 3 presents the scatter of offsets (top), scale values (middle) and non-orthogonalities (bottom) wrt. the results of the analysis of the whole data set (represented by the lines, cf. Table 1). Values for axis 1 are shown with red symbols, while those for axis 2 and 3 are indicated by blue and green color, respectively. The errors (standard deviation) listed in Table 1 are calculated from the scatter of the 174 individual estimates wrt. these mean values (Note that the standard deviation of the individual estimates are $\sqrt{174}=13.2$ times larger than those given in the table).

The upper right plot shows sensor and electronics temperature in red and blue, respectively, and the middle right plot presents the achieved rms misfit for each 4-days segment. Finally, to test for a possible time difference between the two magnetometers, we repeated the calibrations, but allowing for a time-lag $\Delta t$ between the two magnetometers. The right bottom plot of the Figure shows the time-lag for which the smallest rms misfit was achieved. It is believed that (relative) timing errors between the two magnetometers of more than $5 \mathrm{~ms}$ can be identified in this way. From the present analyses there is no indication of a consistent (relative) timing error.

Although the 174 independent estimates of the offsets and scale values generally follow the linear trend given in Table 1 , there are obviously unmodeled signals, perhaps most prominently seen in the scale values of axis 2 (blue symbols) between day count 180 and 540. These signatures may be due to a non-linear drift of the response, due to radiation, aging of the components, or due to other unmodeled dependencies. However, the deviations from the linear trend are below $0.5 \mathrm{nT}$ for the offsets and below $10 \mathrm{ppm}$ for the scale values (corresponding to less than $0.5 \mathrm{nT}$ in a background field of $50000 \mathrm{nT}$ ) and thus close to being negligible.

To summarize: the described in-flight scalar calibration was found to be a stable method to estimate the parameters of the magnetometer response. Since two instruments measuring the magnetic field strength at the same time instant are compared, the method does not require geomagnetic quiet conditions - contrary to the in-flight alignment of the vector magnetometer that is discussed in the next section.

\section{Alignment of the Vector Magnetometer}

Estimation of the remaining 3 parameters - the three Euler angles $\alpha, \beta$ and $\gamma$ describing the rotation from the CSC coordinate system (whose orientation is assumed unknown) to the coordinate system of the SIM (which measures attitude with respect to the celestial coordinate system) - requires knowledge of the magnetic vector $\mathbf{B}_{\mathrm{SIM}}$ in the SIM reference coordinate system. This is achieved by assuming that the magnetic field can be modeled by a scalar potential. Let $\mathbf{T}$ be the rotation matrix that transforms the magnetic vector from a local north, east, center (NEC) spherical coordinate system $\mathbf{B}_{\mathrm{NEC}}=\left(-B_{\vartheta}, B_{\phi},-B_{r}\right)^{T}$ to the vector $\mathbf{B}_{\mathrm{SIM}}=\underline{\underline{\mathbf{T}}} \cdot \mathbf{B}_{\mathrm{NEC}}$ in the coordinate system of the SIM. The matrix $\underline{\overline{\mathbf{T}}}$ is derived from position and from the attitude measurements of the SIM. Using Eq. (3), the relationship between the magnetic vector in the magnetometer coordinate system (calibrated using the parameters found in the previous sections) and the magnetic vector in the NEC coordinate system is given by

$$
\begin{aligned}
\mathbf{B}_{\mathrm{CSC}} & =\underline{\underline{\mathbf{R}}}(\boldsymbol{\epsilon}) \cdot \underline{\underline{\mathbf{T}} \cdot \mathbf{B}_{\mathrm{NEC}}} \\
& =-\underline{\underline{\mathbf{R}}}(\boldsymbol{\epsilon}) \cdot \underline{\underline{\mathbf{T}}} \cdot \operatorname{grad} V
\end{aligned}
$$

with $\boldsymbol{\epsilon}=(\alpha, \beta, \gamma)^{T}$. Here it has been assumed that $\mathbf{B}_{\mathrm{NEC}}=$ $-\operatorname{grad} V$ can be derived from a magnetic scalar potential $V$ which is expanded into series of spherical harmonics. 

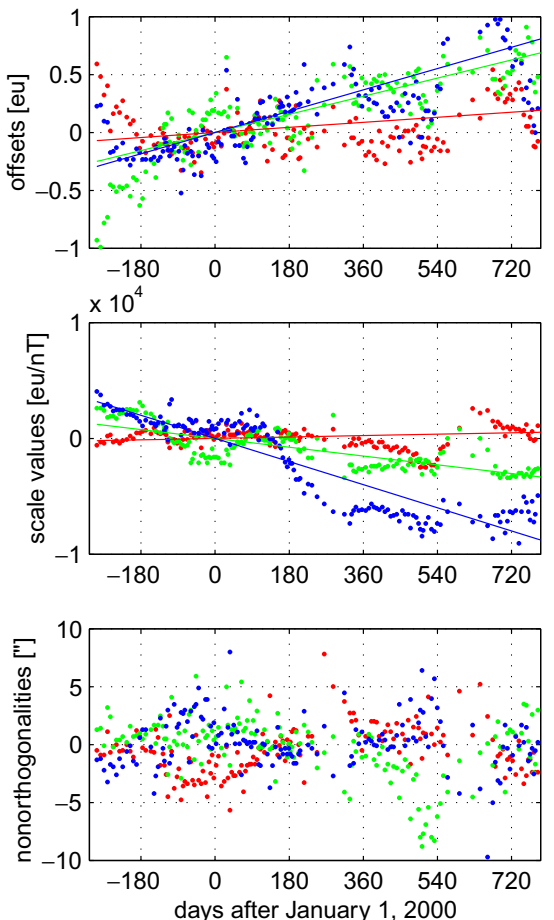
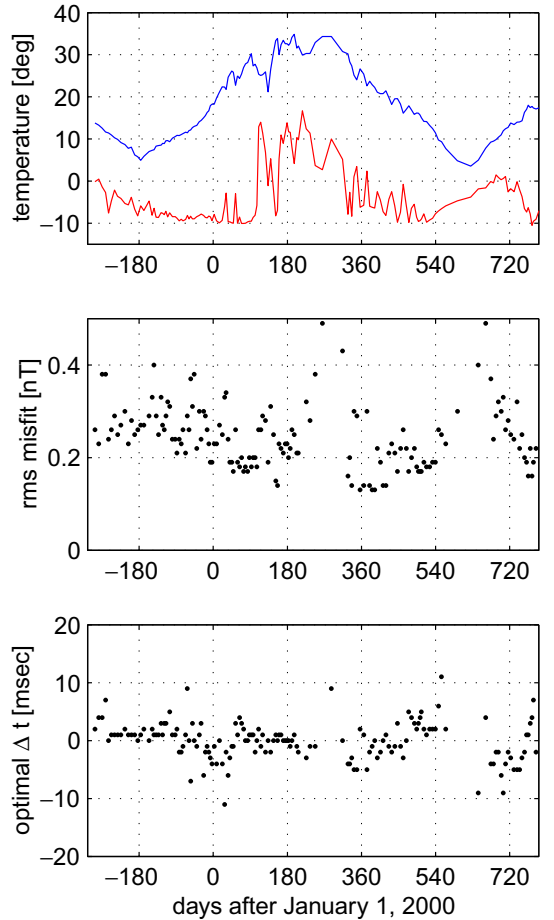

Fig. 3. Left: Scatter of the offsets (top), scale values (middle) and non-orthogonalities (bottom) around the mean values of Table 1 for each of the 174 4-days segments (axis 1: red; axis 2: blue; axis 3: green). Right: Temperature (top, sensor: red; electronics: blue), achieved rms misfit (middle) and optimum time lag between the two magnetometers (bottom).

We applied two methods for the estimation of the Euler angles $\alpha, \beta, \gamma$ : In the first approach we simultaneously solved for the Euler angles and the Gauss coefficients of a field model (up to spherical harmonic degree/order 13), as described in Olsen et al. (2000a). This was the only possible approach at the beginning of the Ørsted mission. However, in the meantime some field models have been estimated using data from Ørsted, CHAMP, Ørsted-2/SAC-C and geomagnetic observatories, which allow us to use a different approach by applying a given field model when estimating the Euler angles rather than estimating the model simultaneously. For this second approach we utilized $\mathbf{B}_{\mathrm{NEC}}$ from the spherical harmonic model of the core and crustal field (up to degree/order 29), of the secular variation (up to degree/order 13), and of the external field (up to degree/order 2, the dipole part of which depends on the (provisional) Dst index of magnetospheric activity) that has been derived from Ørsted data spanning more than two years (March 1999 to September 2001) (Olsen, 2002).

For both approaches we used data segments of typically 1 month length from geomagnetic quiet periods (same selection criteria as used for deriving the model, cf. Olsen et al. (2000a)) and solved for the Euler angles (and, in the first approach, also for the Gauss coefficients of the main field) using a robust least-squares method. Some parameters of the SIM have been changed on January 22, 2000, to improve instrument twist performance. This, as expected, resulted in an apparent change of the optical axis, and hence in a change of the Euler angles of up to 1.5 degrees $^{1}$. We therefore here

\footnotetext{
${ }^{1} \mathrm{~A}$ similar shift of about $1^{\circ}$ was seen between the pre-flight calibration at Table Mountain Observatory (California) in October 1996 and the first inflight calibration, suggesting that this shift may be due to different parameter
}

only report on the results obtained with data between January 22, 2000 and May 2002; see Olsen et al. (2000a) for the in-flight estimation of the Euler angles before January 2000 and Jørgensen (2002) for a description of the pre-flight calibration.

Figure 4 shows the results of both approaches. The mean values of the estimates obtained with the first method (denoted in the Figure with "adjusted field model") are $\alpha=$ $-91.2242^{\circ} \pm 3.9^{\prime \prime}, \beta=-90.1761^{\circ} \pm 2.2^{\prime \prime}, \gamma=+0.4425^{\circ} \pm$ 4.6"; these values (shown with dashed lines in the Figure) differ from the mean values of the second approach (denoted with "fixed field model") by less than 4 arcsec corresponding to less than $1 \mathrm{nT}$ in a field of $50000 \mathrm{nT}$. The probable error amounts to a constant direction bias in the relative orientations of the CSC and the SIM. The difference is largest in $\gamma$ for which the result of the first approach is systematically lower after day count 360, compared to the second approach. In addition, the values around day count 540 (July 2001) are above, while those around day count 720 (January 2002) are below the mean value. We do not believe that these variations are due to a true change in the rotation between the two coordinate systems, but attribute them to magnetic field contributions that are not describable by the chosen parameterization of the scalar potential $V$. A possible explanation for the observed apparent long-term variation of the Euler angles could be the existence of unmodeled local-time dependent magnetic field contributions. As an example: an asymmetric magnetospheric ring-current produces a $B_{\phi}$-component that depends on local time, but (to first order) not on longitude.

settings during pre-flight calibration, too. Investigations are ongoing to establish if the observed shift can be explained by one of a limited number of recorded test parameter sets in use at that time. 

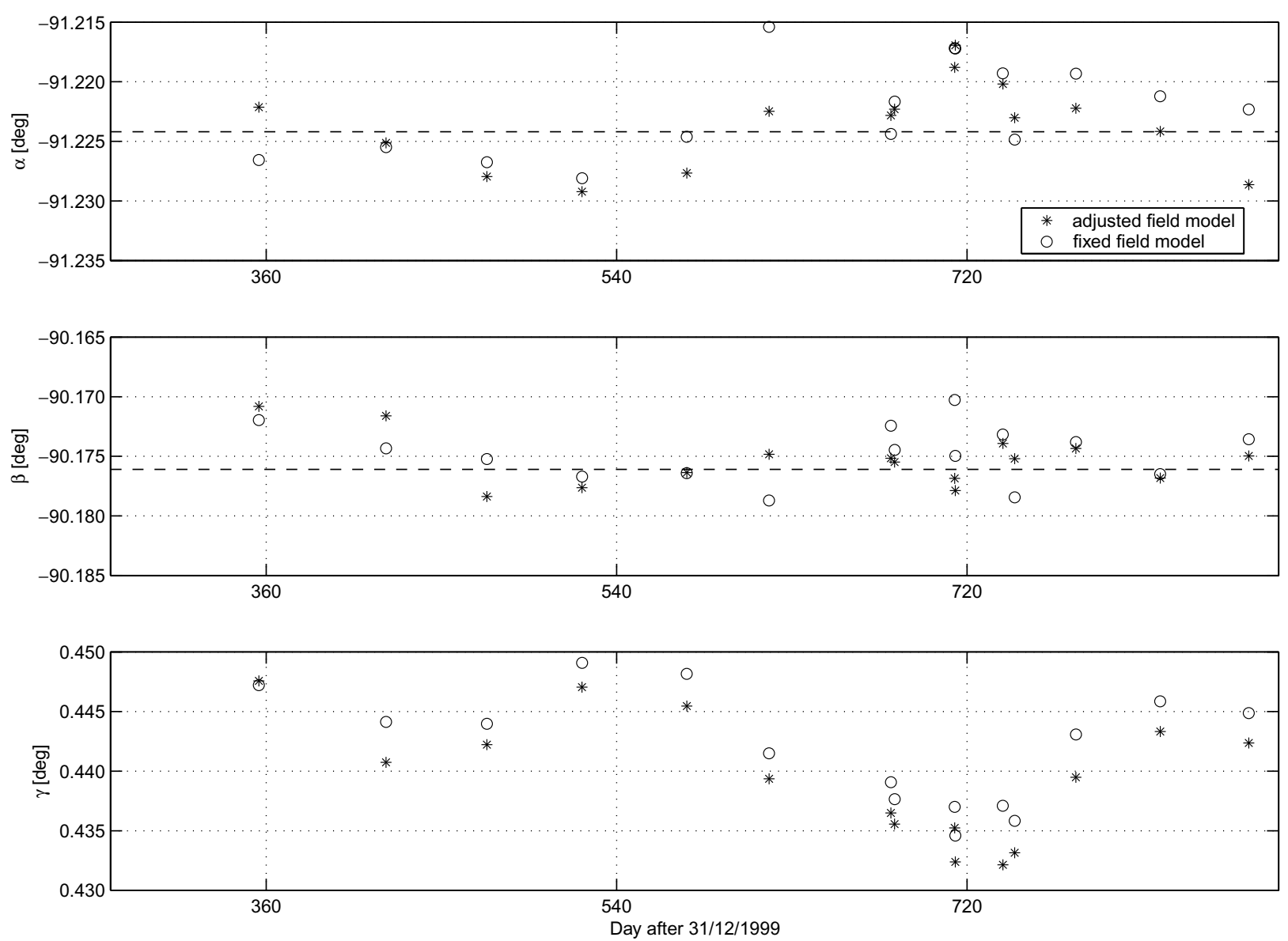

Fig. 4. The Euler angles $\alpha, \beta$ and $\gamma$ describing the CSC-SIM rotation as found from independent data segments between January 2000 and May 2002.

Since the data used here are mostly taken around local midnight, the mean residuals (true $B_{\phi}$ minus $B_{\phi}$ from a geomagnetic field model) will be non-zero (they will only be zero if averaged over all local times, or if the field model includes a proper description of local time contributions). However, a longitude independent $B_{\phi}$ is not compatible with any field model that does not include a local time dependency. Since the mean local time of the used data changed during the mission (from 21:00 in February 2000 over 03:30 in February 2001 to 22:00 in February 2002), possible contributions from local-time dependent magnetic fields may map to an apparent long-period variation of the Euler angles. However, we believe that this influence is minimized by averaging the results of 2 years. The investigation of this effect will be the topic of a future publication.

\section{Conclusions}

We described the method that is used to calibrate the vector fluxgate magnetometer of the Ørsted satellite. It is based on the comparison of the magnetometer output with measurements of the absolute magnetic intensity from the second (scalar Overhauser) magnetometer of the satellite. Using three years of data the agreement between the two magnetometers after calibration is better than $\pm 1 \mathrm{nT}$ for $98 \%$ of the data, and better than $\pm 2 \mathrm{nT}$ for $99.94 \%$ of the data corresponding to less than $0.4 \mathrm{nT}$ rms.

We also determined the transformation between the mag- netometer coordinate system and the reference system of the star imager by comparing the magnetic and attitude measurements with magnetic field values from an independently derived model of the Earth's magnetic field. Using this approach we estimated the Euler angles that describe the transformation with an accuracy of better than 4 arcsec, corresponding to less than $1 \mathrm{nT}$ in a $50000 \mathrm{nT}$ field.

Acknowledgments. We would like to thank Kim Bisgaard for help in data pre-processing, and Richard Holme, Hermann Lühr, Martin Rother, Coerte Voorhies, Mike Purucker and an anonymous referee for fruitful criticism. The Ørsted Project was made possible by extensive support from the Ministry of Trade and Industry, the Ministry of Research and Information Technology and the Ministry of Transport in Denmark. Additional international and crucial support was provided from NASA, ESA, CNES and DARA.

\section{References}

Duret, D., J. Léger, M. Francès, J. Bozom, F. Alcouffe, A. Perret, J. C. Llorens, and C. Baby, Performances of the OVH magnetometer for the danish Ørsted satellite, IEEE Trans. Magn., 32, 4935-4937, 1996.

Gödderz, K., H. Lühr, M. Rother, and R. Bock, CHAMP optical bench star camera/vector magnetometer inter-calibration, Earth Planets Space, 2002 (in preparation).

Hogg, R. V., An introduction to robust estimation, in Robustness in Statistics, edited by R. L. Launer and G. N. Wilkinson, pp. 1-17, Academic Press, San Diego, Calif., 1979.

Jørgensen, J. L., In orbit performance of a fully autonomous star tracker, ESA SP-425, ESTEC, Noordwijk, The Netherlands, 2000.

Jørgensen, P. S., Inter-calibration of accurate attitude instruments, Ph.D. thesis, Oersted.DTU, Technical University of Denmark, 2002. 
Jørgensen, P. S., J. M. G. Merayo, and T. Risbo, A method for the determination of three Euler angles for the SAC-C satellite magnetic mapper probe instrument package, Sensors and Actuators, Sensors and Actuators, A 95, $1-7,2001$.

Lancaster, E., T. Jennings, M. Morrissey, and R. Langel, Magsat vector magnetometer calibration using Magsat geomagnetic field measurements, Techn. Mem. 82046, NASA, 1980.

Merayo, J., P. Brauer, F. Primdahl, J. R. Petersen, and O. V. Nielsen, Scalar calibration of vector magnetometers, Meas. Sci. Technol., 11, 120-132, 2000

Merayo, J. M. G., F. Primdahl, P. Brauer, T. Risbo, N. Olsen, and T. Sabaka, The orthogonalization of magnetic systems, Sensors and Actuators A, 89, 185-196, 2001.

Neubert, T., et al., Ørsted satellite captures high-precision geomagnetic field data, EOS, 82, 81-88, 2001.

Nielsen, O. V., J. R. Petersen, F. Primdahl, P. Brauer, B. Hernando, A Fernandez, J. M. G. Merayo, and P. Ripka, Development, construction and analysis of the 'Ørsted' fluxgate magnetometer, Meas. Sci. Technol., 6, 1099-1115, 1995.

Nielsen, O. V., P. Brauer, F. Primdahl, T. Risbo, J. L. Jørgensen, C. Boe, C. Deyerle, and S. Bauereisen, A high-precision triaxial fluxgate sensor for space applications: Layout and choice of materials, Sensors and Actuators, Actuators, A 59, 168-176, 1997.

Olsen, N., A model of the geomagnetic field and its secular variation for epoch 2000 estimated from Ørsted data, Geophys. J. Int., 149, 454-462, 2002 .
Olsen, N., T. J. Sabaka, and L. Tøffner-Clausen, Determination of the IGRF 2000 model, Earth Planets Space, 52, 1175-1182, 2000a.

Olsen, N., et al., Ørsted initial field model, Geophys. Res. Lett., 27, 3607 3610, 2000b.

Risbo, T. and N. Olsen, Attitude intercalibration of the star imager and the spherical compact sensor magnetometer for the Ørsted geomagnetic satellite mission, SPIE proceedings, 2810, 9, 1996.

Risbo, T., P. S. Jørgensen, and J. Merayo, SAC-C MMP (Ørsted-2) vector magnetometer calibration and camera intercalibration, Tech. rep., Niels Bohr Institute AFG, Juliane Maries Vej 30, DK-2100 Copenhagen Oe, 2001.

Risbo, T., P. Brauer, J. M. G. Merayo, O. Nielsen, J. R. Petersen, F. Primdahl, and N. Olsen., Ørsted calibration mission: The thin shel method and the spherical harmonic analysis, in Ground and In-Flight Space Magnetometer Calibration Techniques, vol. ESA SP-490, 2002 (in press).

Synge, J. L., Classical dynamics, in Handbuch der Physik, edited by S. Flügge, vol. III/1, pp. 1-225, Springer Verlag, Berlin - Göttingen Heidelberg, 1960.

N. Olsen (e-mail: nio@dsri.dk), L. Tøffner-Clausen, T. J. Sabaka, P. Brauer, J. M. G. Merayo, J. L. Jørgensen, J.-M. Léger, O. V. Nielsen, F. Primdahl, and T. Risbo 